\title{
A MACROSCOPIC ANALYSIS OF TRANSPORT NETWORKS: THE INFLUENCE OF NETWORK DESIGN ON URBAN TRANSPORTATION PERFORMANCE
}

\author{
ALI ENES DINGIL ${ }^{1,2}$, FEDERICO RUPI $^{2} \&$ ZANETA STASISKIENE $^{1}$ \\ ${ }^{1}$ Kaunas University of Technology, Institute of Environmental Engineering, Lithuania \\ ${ }^{2}$ University of Bologna, Civil Engineering Department, Italy
}

\begin{abstract}
This research aims at investigating the direct and indirect influence of network structures on urban transportation performance with a macroscopic perspective. Transport systems are complex - the functional properties of a transportation network can affect mobility patterns which in turn changes the network performance. Understanding the topology of transportation networks is important in order to upgrade transport network design and to improve transportation performance. This paper attempts to determine important network indicators such connectivity, centrality and clustering measures for different network types (road, rail and bike) from 86 urban areas and 32 countries, based on comparable, directly observable open-source data such as OpenStreetMap (OSM) and the TomTom congestion database. Relations between indicators are identified through correlation measures. In addition, regression models are calibrated which quantify the relations between infrastructure accessibility (IA) and network indicators and average traffic delay times. The indicator average road connectivity over average road circuity (RCRC), which is proposed in this study, has not been cited before in literature. The main results suggest that the determination of distance-based connectivity of networks is an important proxy to understand road transportation performance. Consequently, two main results were obtained: (1) an increase in average short-distance connectivity of road networks (average closeness centrality and RCRC) eases road congestion, presumably because the network distributes road traffic more homogenously while decreasing low-permeability choke points, (2) an increase of the average short-distance connectivity of networks of alternative modes such as rail or bike (average weighted rail clustering coefficient and average cycle closeness centrality) does alleviate road congestion. In particular, for cities with over $0.4 \mathrm{~km}$ per $\mathrm{km}^{2}$ cycleway density, an increase in cycleway closeness centrality decreases road congestion and it does so almost as efficiently as an increase in road infrastructure accessibility. Presumably, well-connected, alternative networks with short and direct routes convince car users to shift to the alternative mode, which decreases road traffic volumes.
\end{abstract}

Keywords: network design, topology, infrastructure accessibility, congestion, open-source data, OSM, TomTom; transportation performance, transport planning.

\section{INTRODUCTION}

Transportation networks are complex dynamic systems which have been compared with the neural networks of the brain where neural cells distribute information by exchanging chemical transmitters between synapses. [1]. Urban transportation networks are the distributors of cities for energy, materials and people to specific zones of the city, in the same way as a cardiovascular network distributes energy and materials to cells in an organism [2]. The term complexity for transport networks results in a rich behaviour arising from system connections, interactions with subsets and the dynamic processes where vehicles and people act within a network structure (pattern and configuration) [3]. In recent years, understanding the structure and dynamics of urban transport networks has been improved through analyses of network topology measures using the mathematical tools of graph theory [4]. The configuration of networks helps to detect travel behaviours of inhabitants [5, 6], to evaluate transportation performance $[7,8]$ and to understand how cities are organized [1,9]. Complex network 
analysis allows important feedback for urban modelling. It is also an effective evaluation tool since providing feedback of the system is important for correcting, improving or upgrading urban models before executive plans are drafted [10]. Dynamic system variables such as population and traffic volume account for the state of the system as it changes over time [3]. On the other hand, one can evaluate cities through co-evolution, where humans shape their city and are shaped by the city, thus making topological measures an important proxy. Various concepts of graph theory are used to describe network features. Topology of a network can be described as the arrangement (centrality and clustering) and connectivity of a network [11]. Geometric variations of their structure such as shape, density and circuity become more visible when complexity is analysed at a more macroscopic level.

Neglecting the effects of alternative transportation networks (railways and bikeways) is one of the limitations to create general models by means of network analysis [8]. Also, focusing just on local analyses (within cities from same country) would not show certain effects on a worldwide scale. The aim of the present analysis is to investigate the influence of the network configuration of different layers (road, railway and bikeway) on road congestion. A holistic approach has been applied by merging infrastructure accessibility (IA) and network configurations for 86 cities from 32 countries at a macroscopic level. The following section provides a literature review. Network indicators are described and their influence on transportation are summarized. Section 3 motivates data collection for this work and explains the principle data-processing steps and limitations. The analysis and results are presented and discussed in Section 4, while discussions and conclusions in Section 5 interpret and summarize the main findings.

\section{LITERATURE REVIEW}

\subsection{Definition of network design indicators}

Various indicators are identified in the literature as a measure of network patterns. The quality of a transport system can be evaluated based on the intensity of connections between road segments through connectivity measures [12]. There are several indicators to evaluate the connectivity pattern of the networks such as alpha index, beta index, gamma index and eta index [13]. Average node connectivity is a useful network proxy, defined as the average over all pairs of vertices of the maximum number of internally disjoint edges connecting a pair of vertices [14]. It is a measure of network resilience: in networks with low average connectivity, circulation is forced through low-permeability choke points, which increases the risk of traffic jams and network disruptions [3]. Another important indicator is the average circuity of a network (or directness) which is the ratio of shortest distance on the network over the Euclidean distance averaged over all origin-destination pairs in the network [5, 6, 15]. The degree centrality is a local measure which offers a hierarchical view of the city where closeness centrality is mainly radial with a strong side effect. The average degree of centrality is described as an average connection of each road segment to all segments in a network [16]. The average closeness centrality is the average distance of the shortest paths between any node and all other reachable nodes of the network [16]. This captures the notion of accessibility in a city. The average clustering coefficient is a measure of the network structure of nodes, defined as the average number of triangles between each node in a network. The clustering coefficient is a measure of accessibility in terms of directness [10]. 


\subsection{Relationship between network design and travel behaviour}

Some studies have analysed the relationship between network configuration and travel behaviours. Differences of the average network centrality among subzones of cities affect inhabitant life and behaviours through various spatial factors [9]. Eighteen cities across the world have been analysed through multiple centrality assessment by primal geographic network graphs (degree, closeness, betweenness, etc.), and 1-square-mile network comparisons have been conducted [9]. The results have demonstrated that a set of different centrality indices allows capturing the skeleton of most central routes. They are determined by the city structure and subzones which appear to affect spatial cognition and collective dynamical behaviour. Public transit networks are more circuitous than roads, which means that the shortest route is much longer than the line of sight. This is one of the reasons behind the preference of the private auto over public transit [6]. The increase in average circuity of public transit networks can drop transit ridership and can cause a mode shift towards road mobility. Network circuity is also used to explain residential place choice of employers for commuting in US metropolitan cities [5].

\subsection{Relationship between network design and transport performance}

Some works have investigated the role of the network configuration in transportation performance. A positive correlation has been demonstrated between delay time and average circuity of networks and a negative correlation between average circuity and disconnectivity of the networks for 48 cities in the United States [8]. Another paper has compared road transportation performance of the 50 largest metropolitan areas in the United States by comparing hierarchy, connectivity and directness (circuity) of the road networks [7]. The results have shown that a $1 \%$ increase in network connectivity reduces the commute time by $0.1 \%$, a $1 \%$ increase in road accessibility reduces the average metropolitan commute times by $90 \mathrm{~s}$ and a $1 \%$ increase in disconnectivity reduces the auto mode share by $0.061 \%$. The circuity of the network is an important measure of transportation efficiency and is determined by the transport network configuration, transport planning and the underlying terrain. The circuity in turn shapes how inhabitants use urban space for settlement and travel [4]. Circuity of transit networks has been examined for 36 metropolitan areas (excluding the fringes and low accessible zones) in the United States through maps generated by the OpenStreetMap (OSM) [6]. The results have shown that transit circuity exponentially declines as travel time increases, thus helping to understand mode choices. Furthermore, the average circuity of transit networks which is typically greater than the average road circuity in the cities demonstrates how public transit network systems expanded. Networks can be well connected but at the same time poor in terms of directness. Therefore, connectivity and directness can be coupled effectively without impeding each other [17], and in combination they are important measures for road traffic. A recent study [18] has shown that optimizing the closeness centrality and the degree of centrality in a network system reduce travel times and increase route diversity.

\subsection{Relationship between infrastructure dynamics and transport performance}

Unprecedented urbanization generated accelerating demand on mobility systems and put high pressure on urban road networks. As a result, there have been traffic congestion and 
in general a decreasing service level of the roads. Consequently, also fuel consumption and traffic-related air pollutions [19] have increased. A number of studies have found that an increase in road infrastructure increases car ownership and usage [20-22] and an increase in rail network infrastructure increases rail mode share, resulting from a modal shifts from car to public transport [22-]. It has been demonstrated that an increase in demand rises the travel delay time, while an increase in IA (as road length per inhabitant) decreases the travel delay time [25]. Some studies have also shown that an increase in alternative transport systems' infrastructure alleviates traffic road congestion. This effect has been demonstrated in $[25,26]$ for rail transit systems, in [27, 28] for public transportation in general and in [25] for cycling. These results suggest that the IA, in terms of infrastructure per capita, is an important factor for determining the usage of transportation systems. Linking IA and network configuration can help to draw a more general view.

3 Data selection, collection, processing and limitations

\subsection{Infrastructure dynamics-related indicators from cities}

An indicator for the amount of installed infrastructure in a city is the IA, which is expressed as infrastructure length per inhabitant as described in [22]. In this study, IA is chosen to be investigated since it is closely correlated with urban dynamics and transport performance $[22,25]$. Recent population densities and OSM data are used from a previous study [25]: The road infrastructure accessibility (RIA) is expressed in road length per inhabitant, the train infrastructure accessibility (TIA) is expressed in rail length per inhabitant and cycleway infrastructure accessibility (CIA) is expressed in bikeways per inhabitant. As congestion is more challenging for larger cities, this study considers only large cities with over 1 million inhabitants. This selection resulted in a database with 86 cities from 32 countries.

\subsection{Transport performance-related indicator from cities}

The central performance indicator used for this study is the congestion level in terms of average daily extra travel time (ADETT), which is the extra travel time in a day with respect to the free-floating traffic scenario, averaged over all monitored traffic participants of a distinct urban area. Comparable data on the congestion level are retrievable through the TomTom database. TomTom is used by more than 6 million connected GPS devices and traffic is monitored by many million GSM probes and millions of government-owned road sensors [29]. As TomTom's methodology is sufficiently accurate and unified all over the world, it is a suitable data source for the present study. However, errors may occur because the TomTom data are not produced by a representative selection of the population and the special distribution may not be homogeneous.

\subsection{Network design-related indicators from cities}

Several network connectivity measures used in the literature such as alpha index, beta index, gamma index and eta index [13] and average node degree (AND) [30] are selected to investigate. The average closeness centrality is examined here since this indicator is used as overall accessibility of a network and is a good measure of distance-based connectivity (DBC). 
Another distance-based indicator is proposed as the average road connectivity over average road circuity (RCRC). The RCRC is investigated in conjunction with another proxy with regard to traffic congestion. The average weighted clustering coefficient (weighted by edge length) is also considered in the study since an increase in average short triangle connectivity of railways can reduce transit circuity which in turn may impact travel choices.

In literature, comparative topology analyses are generally conducted with samples of the same network size and extracted from mature urban cores. The motivation behind is to compare encapsulated, dense zones in order to make more effective comparison at the same network levels [31]. The threshold: $50 \mathrm{~km}^{2}$ land area [32] for metropolitans is used to justify extraction size of the samples with the bounding box method.

The Python software package OSMnx [30] is used to calculate node and edge numbers of networks and some topological indicators. OSMnx imports any OSM network data of a desired location into a directed transport graph (which is a graph object of the Python networkX package) and allows to perform various topological analyses [33]. OSM is a crowedsourced, unified and publicly available map of the world. OSM infrastructure data look trustworthy for many cities, although it still needs some improvements on micro-level details. However, the OSM data quality is sufficient for macro-level analysis [34]. OSM consists of three basic components: nodes, ways and relations [25]. Each component has various characterizing attributes, called tags. The AND of cities is calculated by OSMnx. Other connectivity measures (alpha, beta, gamma and eta indexes) are calculated as formulated in [13] where node and edge values are provided by OSMnx. The average closeness centrality for roads, railways and cycleways (ARCC, ATCC and ACCC) and the average weighted railway clustering coefficient (AWRCC) is also calculated by OSMnx. A list with all network indicator acronyms is presented in Table 1.

Table 1: Lists of all the network indicator acronyms.

\begin{tabular}{ll}
\hline Average road circuity & $A R C$ \\
Average train circuity & $A T C$ \\
Gamma connectivity & $\gamma$ \\
Beta connectivity & $\beta$ \\
Alpha connectivity & $\alpha$ \\
Eta connectivity & $\eta$ \\
Average node connectivity & $A N C$ \\
Average road connectivity over average road circuity & $R C R C$ \\
Average road closeness centrality & $A R C C$ \\
Average weighted train clustering coefficient & $A W T C C$ \\
Average cycleway closeness centrality & $A C C C$ \\
Road infrastructure m per 10 habitants & $R I A$ \\
Train infrastructure m per 10 habitants & $T I A$ \\
Cycle infrastructure m per 10 habitants & $C I A$ \\
\hline
\end{tabular}




\section{ANALYSES AND RESULTS}

\subsection{Correlations}

The Pearson correlation coefficient between different indicators together with the number of samples is shown in Table 2. The software IBM SPSS 25 is used for the Pearson correlation analyses of variables, while the $95 \%$ confidence level over a 0.2 correlation is taken into account.

Significant negative correlations between ADETT and IA types have already been shown in a previous study, and also regression models between ADETT and RIA have been presented [25]. A considerably negative correlation between road network connectivity indicators and ADETT is seen in Table 1. As expected, the reduction of choke points in the road network can increase continuity of traffic flows and simultaneously reduces traffic congestion. All road connectivity indicators strongly correlated with each other at a similar level; thus, only gamma connectivity is picked to show intercorrelations between connectivity measures. Only eta connectivity did not show any considerable correlation with any other indicators. Average circuity of road and rail networks (ARC and ATC) is positively correlated with ADETT. As expected, network connectivity is negatively correlated with network circuity for both network types.

Networks can be well connected but at the same time be poor in terms of directness. This confirms that connectivity and directness are independent and can be coupled effectively as suggested in [17]. Table 3 demonstrates the relation between distance-based network

Table 2: Pearson correlation coefficient and the number of samples $(\mathrm{N})$ between different indicators.

\begin{tabular}{|c|c|c|c|c|c|c|c|c|c|c|}
\hline Correlation & n ADETT & RIA & TIA & CIA & $\gamma$ & $\beta$ & $\alpha$ & ANC & ARC & ATC \\
\hline ADETT & & $\begin{array}{c}N=87 \\
-0.693\end{array}$ & $\begin{array}{c}N=85 \\
-0.408\end{array}$ & $\begin{array}{c}N=86 \\
-0.324\end{array}$ & $\begin{array}{c}N=87 \\
-0.490\end{array}$ & $\begin{array}{c}N=87 \\
-0.490\end{array}$ & $\begin{array}{c}N=87 \\
-0.490\end{array}$ & $\begin{array}{c}N=87 \\
-0.490\end{array}$ & $\begin{array}{l}N=87 \\
0.286\end{array}$ & $\begin{array}{l}N=87 \\
0.388\end{array}$ \\
\hline$\Gamma$ & & & & & $\begin{array}{l}N=87 \\
0.991\end{array}$ & $\begin{array}{l}N=87 \\
0.991\end{array}$ & $\begin{array}{l}N=87 \\
0.991\end{array}$ & $\begin{array}{l}N=87 \\
0.950\end{array}$ & & \\
\hline ARC & $\begin{array}{l}N=87 \\
0.286\end{array}$ & & & & $\begin{array}{c}N=87 \\
-0.363\end{array}$ & $\begin{array}{c}N=87 \\
-0.363\end{array}$ & $\begin{array}{c}N=87 \\
-0.363\end{array}$ & $\begin{array}{c}N=87 \\
-0.363\end{array}$ & & \\
\hline
\end{tabular}

Table 3: Pearson correlation coefficient and number of samples $(N)$ between different indicators.

\begin{tabular}{lllll}
\hline Correlation & RCRC & ARCC & AWTCC & ACCC \\
\hline ADETT & $N=87,-0.503$ & $N=87,-0.385$ & $N=54,-0.466 N=29,-0.445$ \\
$\Gamma$ & $N=87,0.991$ & $N=87$ & 0.425 & \\
ARC & $N=87,-0.482$ & $N=87,-0,402$ & \\
ATC & & & $N=54,-0.356$ \\
RCRC & & $N=87,0.456$ & \\
\hline
\end{tabular}


connectivity measures (ARCC, ATCC and ACCC) and ADETT with some interrelations. Average closeness centrality is negatively correlated with ADETT for road and cycle networks (ARCC and ACCC). Another distance-based indicator proposed here as 'average road connectivity over average road circuity (RCRC)' is correlated with ADETT with a coefficient of -0.503 . As ARCC and RCRC are similar type of measures, they are strongly correlated with each other. Presumably, a high average short-distance accessibility of road networks decreases low-permeability choke points and distributes road traffic more homogenously and consequently eases road traffic. The average weighted clustering coefficient demonstrates distance-based directness of the network, as seen by the fact that AWTCC correlates negatively with ADETT with a coefficient of 0.466 and negatively correlates with the average rail circuity.

\subsection{Comparative view}

Road network connectivity maps of some cities $\left(50 \mathrm{~km}^{2}\right.$ cores $)$ are plotted with the dual graph approach where streets are represented as nodes and intersections are represented as edges in Figs 1-2. These pairs of cities were selected for comparison as each pair has similar peer sociodemographic and infrastructure indicators, as shown in Table 4. Their similarity can allow putting in evidence the influence of network structure. Qualitative colour maps increasing luminance through blue, purple and yellow hues where node connectivity decreases from
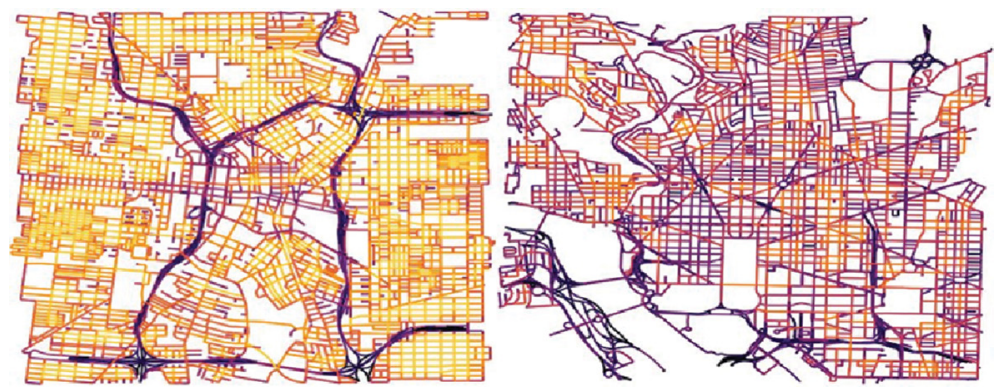

Figure 1: Road network connectivity maps of San Antonio and Washington.
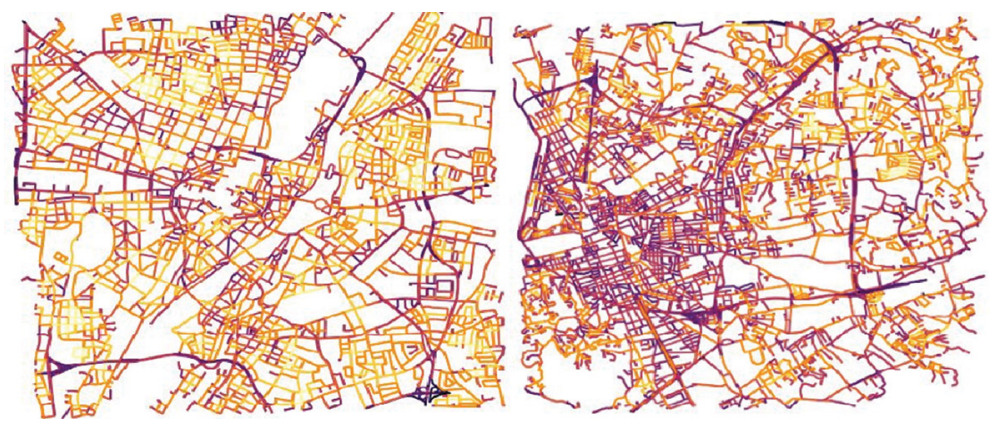

Figure 2: Road network connectivity maps of Berlin and Marseille. 
Table 4: Comparison of selected cities.

\begin{tabular}{lccccccccc}
\hline Cities & RIA & TIA & CIA & $\begin{array}{c}\text { ADETT Population } \\
\text { density }\end{array}$ & ARC & ANC & $\Gamma$ & RCRC \\
\hline San Antonio & 60.309 & 2.613 & 0.387 & 26 & 1,312 & 1.0216 & 5.887 & 0.9817 & 0.9609 \\
Washington & 57.105 & 1.893 & 0.344 & 33 & 1,446 & 1.0284 & 4.932 & 0.8225 & 0.7998 \\
Dublin & 24.645 & 1.453 & 0.262 & 50 & 3,716 & 1.0736 & 3.945 & 0.6579 & 0.6128 \\
Manchester & 24.021 & 1.643 & 0.115 & 44 & 4,236 & 1.0469 & 4.698 & 0.7832 & 0.7481 \\
Berlin & 18.550 & 6.477 & 0.080 & 28 & 3,700 & 1.0276 & 5.032 & 0.8392 & 0.8166 \\
Marseille & 17.651 & 3.177 & 0.025 & 41 & 4,040 & 1.0608 & 4.229 & 0.7050 & 0.6646 \\
\hline
\end{tabular}

glossy yellow to matte purple are conducted to interpret the graphs more efficiently. It is clearly seen in plotted maps and in Table 4 that cities with higher RCRC and higher road network connectivity perform lower congestion.

\subsection{Statistical models}

As ADETT and distance-based road connectivity measures (DBRCs) are considerably correlated, some statistical models have been calibrated with the entire set of cities. ADETT as a function of DBRC appears to be of an exponential shape:

$$
A D E T T=a \exp (b D B R C)
$$

However, the fitting errors with a linear model is only slightly superior. Figure 3 demonstrates the exponential model which has been calibrated from the exponential relation of eqn (1). The best-fitting exponential curve is also shown in Fig. 3. Even though $R_{2}=0.2812$ is not particularly high, it shows a reasonable goodness of fit and parameters are statistically significant. Note that, a similar reasonable fit has also been achieved for ADETT as a function of ARCC. Considering the fact that many different network attributes influence the demand and road congestions, the quality of this fit is reasonable. The influence of DBRC on congestion is minor for cities with very high population densities such as Bangkok and Mexico (see Fig. 3).

The question is how the road-based coefficients behave in cities in the presence of different alternative infrastructures such as railways and cycleways of different length and topology. Geometric variations of network structures such as density and circuity become more visible when network complexity is at a maturated level [11]. This makes infrastructure density a good proxy to identify the level of infrastructure maturity. Two subsets of cities are created based on the level of maturity of alternative network systems. The rail infrastructure density division has been set at $1 \mathrm{~km}$ per $\mathrm{km}^{2}$ and cycle infrastructure density division has been set at $0.4 \mathrm{~km}$ per $\mathrm{km}^{2}$. Both thresholds have been chosen arbitrarily. The main idea has been to isolate non-matured alternative network systems in cities. This will help to understand network-related factors of alternative network systems more accurately. All cities possess a road network with a density over $10 \mathrm{~km}$ per $1 \mathrm{~km}^{2}$. As IA and ADETT as well as DBC and ADETT are considerably correlated, some multiple linear regression models were attempted with the entire set of cities as well as on specific subsets (cities matured with railways -53 cities and cities matured with cycleways -28 cities), while only the models with a $P$ value 


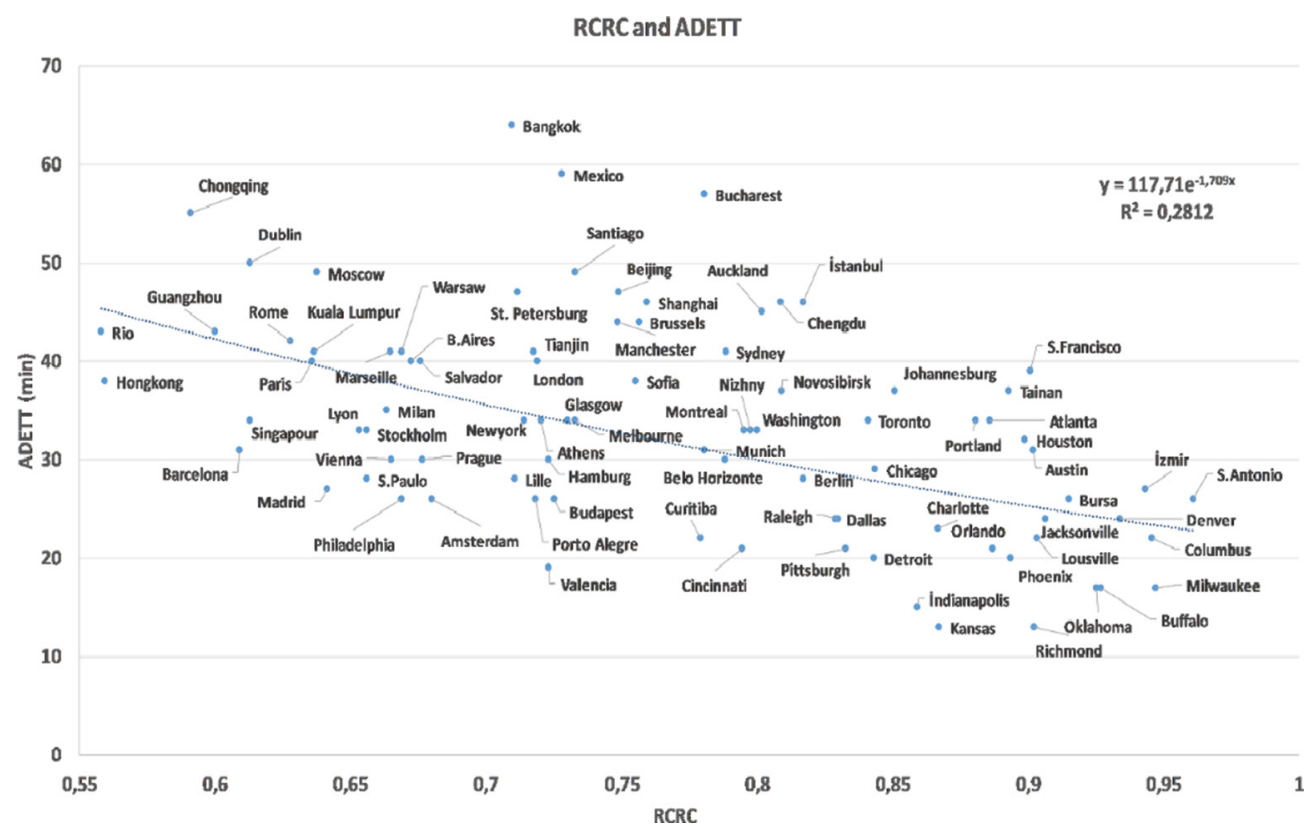

Figure 3: Scatter graph with congestion level (ADETT) of cities over RCRC; the dotted line represents the fitted exponential curve from Eq.(1).

below 0.2 for each attribute are shown in the tables below. Further models were built, which include IA indicators (infrastructure per capita) and DBC indicators:

$$
\begin{gathered}
A D E T T=c+d R I A+e T I A+f A R C C \\
A D E T T=c+d R I A+e T I A+f A R C C+g A W T C C \\
A D E T T=c+d R I A+e T I A+f C I A+g A C C C
\end{gathered}
$$

Tables 5-7 demonstrate the calibration results of the linear function model eqns $(2-5)$. The coefficients $d, e, f$ and $g$ quantify the reduction in traffic congestions due to an increase/ decrease of the independent variables. As units are different, standardized beta coefficients are considered. The results of the linear function model from eqn (2) demonstrate that an increase in average road closeness centrality reduces congestion at a similar level as does an increase in TIA. This is a useful finding which demonstrates that network design is as important as increasing the infrastructure length of alternative networks. The significance for $e, f$ and $g$ is less clear. However, the results of the linear function model (eqn (3)) demonstrate that an increase in AWTCC decreases road congestion at a similar level as does an increase in average road closeness centrality. The results of the linear function model (eqn (4)) demonstrate that the influence of RIA on congestion stays a little lower compared with model eqn (3) and CIA has the highest influence on congestion alleviation compared with an increase in RIA and TIA, while considering cities with over $0.4 \mathrm{~km}$ per km${ }^{2}$ cycleway density. Furthermore, an increase in $A C C C$ decreases congestion more effectively than an increase in TIA. However the increase in $A C C C$ is slightly less effective in decreasing congestion than an increase in RIA. The influence of TIA is less significant in this model. 
Table 5: Calibration results of the linear function model eqn (2) for all samples. $R^{2}=0.527$, sample size $N=85$.

\begin{tabular}{llrrrr}
\hline Calibration results & Coefficient & Std Err & Beta & $t$ & $P>|t|$ \\
\hline$c$ & 68.192 & 10.034 & & 6.796 & 0.000 \\
$d$ & -0.222 & 0.034 & -0.556 & -6.603 & 0.000 \\
$e$ & -0.682 & 0.312 & -0.179 & -2.186 & 0.032 \\
$f$ & $-109,818.322$ & 46,148 & -0.193 & -2.380 & 0.020 \\
\hline
\end{tabular}

Table 6: Calibration results of the linear function model Eq. (3) for cities with mature railways. $R^{2}=0.482$, sample size $N=53$.

\begin{tabular}{llccrr}
\hline Calibration results & Coefficient & Std Err & Beta & $t$ & $P>|t|$ \\
\hline$c$ & 71.287 & 16.076 & & 4.434 & 0.000 \\
$d$ & -0.161 & 0.041 & -0.488 & -3.926 & 0.000 \\
$e$ & -0.847 & 0.423 & -0.206 & -2.002 & 0.051 \\
$f$ & $-121,553.58$ & 72,325 & -0.180 & -1.681 & 0.099 \\
$g$ & $-1,017.277$ & 764.075 & -0.164 & -1.331 & 0.189 \\
\hline
\end{tabular}

Table 7: Calibration results of the linear function model eqn (4) for cities with mature cycleways. $R^{2}=0.593$, sample size $N=28$.

\begin{tabular}{llcccc}
\hline Calibration results & Coefficient & Std Err & Beta & $t$ & $P>|t|$ \\
\hline$c$ & 46.366 & 2.803 & & 16.163 & 0.000 \\
$d$ & -0.167 & 0.054 & -0.461 & -3.039 & 0.006 \\
$e$ & -0.684 & 0.393 & -0.229 & -1.571 & 0.130 \\
$f$ & -2.833 & 0.797 & -0.472 & -3.486 & 0.002 \\
$g$ & $-20,519.767$ & 9563 & -0.321 & -2.127 & 0.044 \\
\hline
\end{tabular}

\section{DISCUSSIONS AND CONCLUSIONS}

In the past, only limited holistic analysis have attempted to investigate the influence of urban network topology on transportation performance mainly due to a lack of diversity in the data from alternative network types. We analysed the influence of the topological indicators with multinetwork layers on network performance. The 86 analysed cities show diversity, as they are distributed over 32 countries.

Relationships between network topology indicators and congestion level have been investigated. The infrastructure data have been taken from the open-source OSM and traffic-related data from the TomTom database. Open-source data-related errors and data limitations have been highlighted. Good correlation values between topological variants and congestion levels have been demonstrated. Multiple linear regression models were attempted with the entire set of cities as well as on specific subsets (53 cities with mature railways and 28 cities with 
mature cycleways). Calibrated regression models were proposed, quantifying the relation between transport infrastructure, topology and performance indicators.

The core message of the study is to demonstrate the influence of network design-related factors on road traffic performance. The findings suggest that short-distance connectivity of the road network is important for reducing traffic congestion. Another hypothesis is that DBC of alternative networks does influence travel mode choices which in turn changes road traffic volume. One particular question has been addressed with regard to urban planning: what is more sustainable in congestion alleviation, building well-connected road networks with low circuity or building well-connected alternative networks with low circuity? Public transit networks are more circuitous than roads, which is likely to be one of the reasons why people prefer the auto usage over public transit [6].

The main result is that the DBC of networks is an important proxy to understand road transportation performance. Following this reasoning, one can draw two main conclusions: (1) an increase in average short-distance connectivity of road networks (average closeness centrality and RCRC) eases road congestion, most likely because the road traffic is distributed more homogenously over a network with less low-permeability choke points and (2) an increase in average short-distance connectivity of alternative network systems (average weighted rail clustering coefficient and average cycle closeness centrality) alleviates road congestion. In particular, for cities with a mature cycleway network (cycleway density $>0.4$ $\mathrm{km}$ per $\mathrm{km}^{2}$ ), an increase in cycleway closeness centrality decreases congestion with nearly the same effectiveness as an increase in RIA. Subsequent cost studies could identify which network modification would be the most cost efficient, in average. Presumably, well-connected alternative networks with short direct routes can trigger a mode shift from car to rail or bike, thus lightening road traffic volumes. Furthermore, when alternative network systems are mature, the impact of road infrastructure indicators on transportation performance is less important. With the available average daily travel distance data for each mode, the study could be further exploited to determine the relationship between DBC indicators and travel distance.

\section{REFERENCES}

[1] Newman, M.E.J., Networks: An Introduction. Oxford University Press, 2010.

[2] Samaniego, H. \& Moses, M.E., Cities as organisms: Allometric scaling of urban road networks. Journal of Transport and Land Use, 1, pp. 21-39, 2009.

[3] Boeing, G., Measuring the complexity of urban form and design. Urban design international. 2018a. https://doi.org/10.1057/s41289-018-0072-1.

[4] Boeing, G., The morphology and circuity of walkable and drivable street networks. SSRN Electronic Journal. 2018b. 10.2139/ssrn.3119939.

[5] Levinson, D.M. \& El-Geneidy, A., The minimum circuity frontier and the journey to work. Regional Science and Urban Economics, 39(6), pp. 732-738, 2009.

[6] Huang, J. \& Levinson, D.M., Circuity in urban transit networks. Journal of Transport Geography, 48, pp. 145-153, 2015.

[7] Levinson, D., Network structure and city size. Plos One, 7, pp. 1, 2012.

[8] Parthasarathi, P., Network structure and metropolitan mobility. Journal of Transport and Land Use, 7(2), pp. 153-170, 2014. https://www.jtlu.org/index.php/jtlu/article/ view/494.

[9] Crucitti, P., Latora, V. \& Porta, S., Centrality in Networks of Urban Streets, vol. 16, Chaos, pp. 1, 2006. 
[10] Tsiotas, D. \& Polyzos, S., The Topology of Urban Road Networks and its Role to Urban Mobility, vol. 24, Transportation Research Procedia, pp. 482-490, 2017.

[11] Xie, F. \& Levinson, D., Measuring the structure of road networks. Geographical Analysis, 39, pp. 336-356, 2007.

[12] Sreelekha, M., Krishnamurthy, K. \& Anjaneyulu, M., Assessment of topological pattern of urban road transport system of Calicut city. Transportation Research Procedia, 17, pp. 253-262, 2016.

[13] Kansky, K., Structure of transportation networks: Relationships between network geometry and regional characteristics. Research paper no. 84, Ph. D. thesis, University of Chicago, 1963

[14] Beineke, L.W., Oellermann, O.R. \& Pippert, R.E., The average connectivity of a graph. Discrete Mathematics, 252, pp. 31-45, 2002.

[15] Barthelemy, M., Spatial networks. Physics Reports, 499(13), pp. 1-101, 2011

[16] Jayaweera, Nadeesha, Perera, K.K.K.R. \& Munasinghe, Jayantha, Centrality measures to identify traffic congestion on road networks: A case study of Sri Lanka. IOSR Journal of Mathematics, 13, pp. 13-19, 2017.

[17] Derrible, S. \& Kennedy, C., Characterizing metro networks: state, form, and structure. Transportation, 37, pp. 275-297, 2010.

[18] Carpio-Pinedoa, José, Martínez-Conde, Javier Aldecoa \& Daudéna, Francisco Lamíquiz, Mobility and urban planning integration at city-regional level in the design of urban transport interchanges. Procedia Social and Behavioral Sciences, 160, pp. 224-233, 2014.

[19] Wang, P, Hunter, T., Bayen, AM., Schechtner, K \& González, MC., Understanding road usage patterns in urban areas. Nature, Scientific Reports, 1001, pp. 2, 2012.

[20] Ingram, G. \& Liu, Z., Determinants of motorization and road provision. Essays in Transportation Economics and Policy, ed. Gomez Ibanez et al., Brookings Institution Press, pp. 325-356, 1999.

[21] Melo, P.S., Graham, D J. \& Canavan, S., Effects of Road Investments on Economic Output and Induced Travel Demand Evidence for Urbanized Areas in the United States, vol. 2297, Transportation Research Record, pp. 163-171, 2012.

[22] Dingil, A.E., Schweizer, J., Rupi, F. \& Stasiskiene, Z. Updated models of passenger transport related energy consumption of urban areas. Sustainability, 11, pp. 4060, 2019. https://doi.org/10.3390/su11154060.

[23] Hass-Klau, C., Crampton, G., Biereth, C. \& Deutsch, V., Bus or Light Rail: Making the Right Choice: A Financial, Operational, and Demand Comparison of Light Rail, Guided Busways and Bus Lanes. Brighton and Government of United Kingdom: Environmental \& Transport Planning, 2004.

[24] Urban Transportation Group, Rail Cities UK: Our vision for their future. Report. 2018. Retrieved from: http://www.urbantransportgroup.org/resources/types/reports/rail-cities-uk-our-vision-their-future.

[25] Dingil, A.E., Schweizer, J., Rupi, F. \& Stasiskiene, Z., Transport indicator analysis and comparison of 151 urban areas, based on open source data. European Transport Research Review (ETRR), 10, pp. 58, 2018. https://doi.org/10.1186/s12544-018-0334-4.

[26] Bhattacharjee, S. \& Goetz, A.R., Impact of light rail on traffic congestion in Denver. Journal of Transport Geography, 22, pp. 262-270, 2012. 
[27] Nelson, P., Baglino, A., Harrington, W., Safirova, E. \& Lipman, A., Transit in Washington, D.C.: Current benefits and optimal level of provision. Journal of Urban Economics, 62(2), pp. 231-251, 2007.

[28] Anderson, M.L., Subways, strikes and slowdowns: The impact of public transit on traffic congestion. American Economic review, 104(9), pp. 2763-2796, 2014.

[29] TomTom, Real time \& historical traffic: TomTom delivers a unique proposition. 2018. Retrieved from: https://www.tomtom.com/lib/doc/licensing/RTTHT.EN.pdf.

[30] Boeing, G., OSMnx: New methods for acquiring, constructing, analyzing, and visualizing complex street networks. Computers Environment and Urban Systems, 65, pp. 126-139, 2017.

[31] Oliveira, E.A, Furtado, V., Andrade, J.S. \& Makse, H.A., Worldwide model for boundaries of urban settlements. Royal Society Open Science, 5, 2018. http://doi.org/10.1098/ rsos. 180468.

[32] Kirkley, A., Barbosa, H., Barthelemy, M. \& Ghoshal, G., From the betweenness centrality in street networks to structural invariants in random planar graphs. Nature Communications, Nature Publishing Group, 2018, 9, pp. 2501, 2018.

[33] Dingil, A.E., Schweizer, J., Rupi, F. \& Stasiskiene, Z., Road network extraction with OSMNx and SUMOPy. SUMO 2018-Simulating Autonomous and Intermodal Transport Systems, 2, pp. 111-117, 2018. https://doi.org/10.29007/t7pk.

[34] Basiri, A., Mike, J., Pouria, A., Amir, P., Monika, S., Adam, W., Terry, M. \& Lijuan, Z., Quality assessment of OpenStreetMap data using trajectory mining. Geo-spatial Information Science, 19(1):56-68, 2016. 